\title{
The Development of Remote Laboratory Sessions at the Stability Wind Tunnel of Virginia Tech During the Coronavirus Pandemic
}

\author{
Máté Szőke ${ }^{1}$, Aurélien Borgoltz ${ }^{2}$, Matthew Kuester ${ }^{3}$, \\ Nanyaporn Intaratep ${ }^{4}$, William Devenport ${ }^{5}$ \\ Center of Research in Experimental Aero/hydrodynamic Technology (CREATe), \\ Kevin T. Crofton Department of Aerospace and Ocean Engineering, \\ Virginia Tech, Blacksburg, VA 24061 \\ Andrew Katz ${ }^{6}$ \\ Department of Engineering Education, \\ Virginia Tech, Blacksburg, VA 24061
}

\begin{abstract}
This paper discusses the remote delivery of wind tunnel experiments performed at the Stability Wind Tunnel of Virginia Tech, in April 2020, during the early stages of the coronavirus pandemic. The originally in-person laboratories were transformed to entirely remote sessions, on a time-frame of a few weeks, to ensure the delivery of the laboratory sessions and the safety of all participants via social distancing and the use of widely-available video conferencing software. The paper outlines the structure of the laboratory sessions, comprising the tour of the facility, data acquisition, and data visualization alongside with all information technology components used to ensure the successful remote delivery of the laboratory sessions. After the two-week-long experimental campaign, participating students provided feedback on the efficacy of the laboratories via a detailed questionnaire. It was found that the students were highly satisfied with the remote delivery of the laboratory sessions but showed a preference for in-person laboratories.
\end{abstract}

\section{Introduction}

Laboratory sessions (or labs) are an essential part of scientific education, particularly in the field of engineering, where students are provided the opportunity to perform experiments related to their studies. Unfortunately, running laboratory teaching experiments are associated with high expenses [1]. They also impose a high load on the laboratories because they are often set up to meet research and not teaching needs. Covering the expenses of labs, namely, instrumentation, maintenance, and instructors, often place significant financial loads on departments and colleges.

Alternative solutions exist to deliver labs to students. The two main alternatives are remote labs and simulated labs. In remote labs, students are physically separated from the laboratory space and they remotely access the

\footnotetext{
${ }^{1}$ Senior Research Associate, Department of Aerospace and Ocean Engineering, AIAA Member, email: $\underline{\text { m.szoke@ vt.edu }}$

${ }^{2}$ Research Associate Professor and Assistant and Director of Virginia Tech Stability Wind Tunnel, Department of Aerospace and Ocean Engineering, AIAA Member

${ }^{3}$ Research Assistant Professor, Aerospace and Ocean Engineering

${ }^{4}$ Research Assistant Professor, Aerospace and Ocean Engineering

${ }^{5}$ Professor and Director of Virginia Tech Stability Wind Tunnel,

Department of Aerospace and Ocean Engineering, AIAA Associate Fellow

${ }^{6}$ Assistant Professor, Department of Engineering Education
} 
instrumentation via computer screens, while in simulated labs, the laboratory space and equipment are emulated using virtual environments [2]. The main advantages of these approaches are reduced costs, flexibility in terms of safety and scheduling. From the student's perspective, however, they provide a reduced experience [3]. While the advantages could be considered minor from a student's perspective, the study of Ma and Nickerson [4] states that the nature of the lab session also dictates which delivery method (hands-on, remote, or simulated) is best suitable for a certain experiment [5]. For example, remote or simulated labs could be a better fit for experiments involving serious health and safety hazards (such as operating high-energy lasers). Furthermore, even in the case of hands-on experiments, the students most often control various computer software to manipulate test conditions, therefore, the conventional definition of a hands-on experiment is less often ensured even in traditional in-person laboratories.

During the spring semester of 2020, the coronavirus pandemic brought a fundamental disruption to higher educations. As a result, universities transitioned to on-line curriculum delivery to ensure the safety and wellbeing of students and faculty. By the end of March 2020, almost all universities over the world were operating entirely online. In many cases, laboratory experiments were simply discontinued, mainly due to the urgency to respond to the situation. However, with minor upgrades to pre-existing hands-on labs, remote laboratory sessions could be considered a reasonable alternative to sustain lab delivery. While during the early months of the coronavirus pandemic, many assumed that life will return to normal in a matter of weeks or months, by the summer of 2020 universities and governments came to realize that social distancing must remain in effect for extended periods of time as higher educations continued on-line operation during the 2020-2021 academic year. These conditions suddenly impose a high demand on the development of remote laboratory sessions.

The response of Virginia Tech (VT) to the coronavirus pandemic was also to transition to on-line education. At VT, the Stability Wind Tunnel (SWT) fulfils important educational responsibilities by accommodating undergraduate laboratories for several weeks during each semester. The SWT also serves as an important industrial test facility for external customers and it also accommodates high-fidelity research experiments [6]-[10]. Due to its large size on the scale of university-based wind tunnels and the efficiency dictated by industrial tests, the experiments at the SWT are operated from a room separated from its test section. This creates a favorable condition to convert the hands-on undergraduate laboratory sessions held at the SWT to remote experiments. Laboratory sessions at the SWT are often combined with research experiments as this provides cost reductions and also brings students closer to cutting-edge research. In April 2020, the scheduled lab and research experiments were focusing on studying trailing edge noise generation of a 3-ft chord NACA0012 airfoil [11], [12] via measuring the far field self-noise of the airfoil under various conditions. As an alternative response to canceling the labs, a decision was made to transition to remote delivery of these laboratory sessions.

In this paper, we will present how the remote delivery of the laboratory sessions was developed, over the course of a few weeks, during the coronavirus pandemic at the SWT [13]. We will present all components that were necessary to the successful delivery of the hybrid laboratory sessions, in particular, all computers, streaming devices, participants in the on-line laboratory sessions will be introduced and discussed. After the two-week-long teaching campaign, the participating students were asked to fulfil a survey, from which results will also be shown. The majority of the components used at the SWT to deliver the laboratory sessions are already available at other universities, therefore, the effort presented here can be a good basis for transitioning to on-line laboratory delivery at other universities either during the COVID-19 pandemic and also in the future.

\section{Experimental description}

The laboratory experiments presented in this paper were performed in the Stability Wind Tunnel at Virginia Tech. The experiments focused on the measurement of airfoil trailing edge noise. In particular, the trailing edge noise was captured using far field microphones, while surface pressure microphones were also embedded in the model in the close vicinity of the trailing edge. The airfoil was equipped with trailing edge add-ons of various shapes to mitigate its trailing edge noise generation. The students were responsible to analyze the acoustic data obtained using the far field and surface pressure microphones. In addition, a high-speed particle image velocimetry (PIV) system was also used to visualize and capture the velocity field within the boundary layer as it passes over the trailing edge of the airfoil. From the instantaneous velocity field, the mean velocity profile was provided to the students for further analysis. In the following, we review the different instruments used in these experiments. 


\section{A. The Stability Wind Tunnel}

The Stability Wind Tunnel is a low-speed closed-circuit wind tunnel used for a mix of commercial testing, research, and educational experiments on the campus of Virginia Tech in Blacksburg, Virginia, see Fig. 1. The facility can generate flow speeds of up to $80 \mathrm{~m} / \mathrm{s}$ through its $7.3 \mathrm{~m}$ long $1.85 \mathrm{~m}$ square test section. Flow through the empty test sections is closely uniform and of very low turbulence intensity, increasing from $0.021 \%$ at $21 \mathrm{~m} / \mathrm{s}$ to $0.031 \%$ at $57 \mathrm{~m} / \mathrm{s}$. Two interchangeable test sections are available in the wind tunnel. The hard wall "aerodynamic" test section has walls formed from a sequence of aluminum panels. Test objects, such as airfoils, are mounted $3.56 \mathrm{~m}$ downstream of the test section entrance. A total of 228 pressure taps are mounted in the contraction and side walls of the test section, and they are used for monitoring the wall boundary condition. The Kevlar-wall "aeroacoustic" test section replicates the same interior dimensions as the hard wall test section, and it is primarily used for aeroacoustic testing as it has two anechoic chambers flank to the test section. The Kevlar walls enable acoustic investigation of test objects as this material is near-impermeable to flow and has a minimal acoustic transmission loss. The Kevlar-walled aeroacoustic test section was used in this study to enable the measurement of the far-field self-noise of the NACA0012 airfoil.

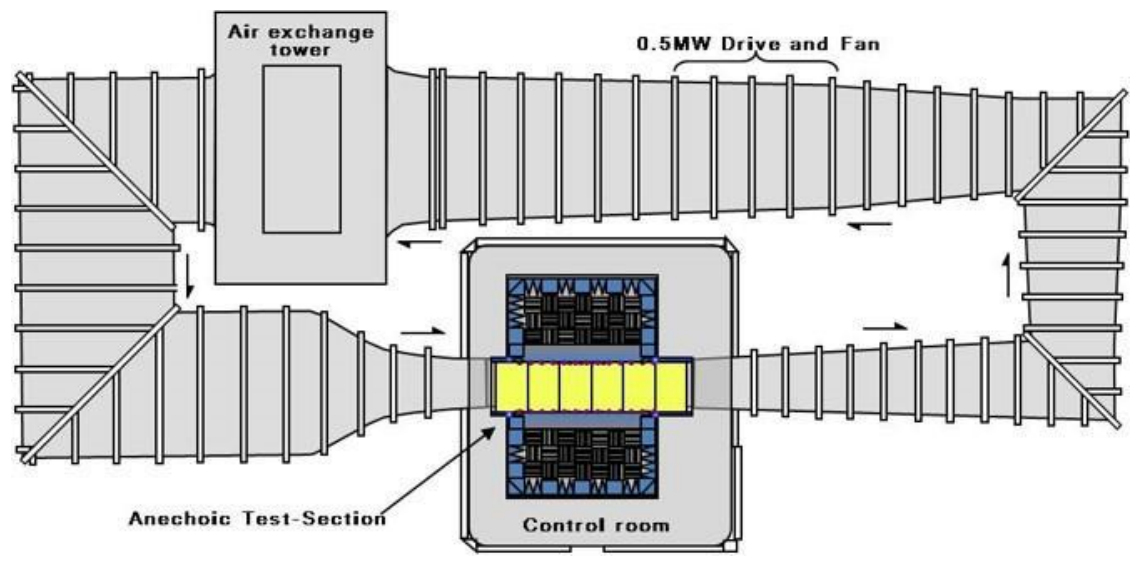

Figure 1: The Stability Wind Tunnel.

\section{B. Experimental apparatus}

\section{The NACA0012 airfoil}

The airfoil model used in this experiment is the uncambered symmetrical NACA0012 airfoil section with $0.914 \mathrm{~m}$ (3 feet) chord and 1.85m (6 feet) span covering the complete vertical height of the test section. The model inside the test section is shown in Figure 2. In this experiment, the airfoil was kept at a 0-degree geometrical angle of attack (AoA) for all tests. The model was instrumented with approximately 80 pressure taps of $0.5 \mathrm{~mm}$ internal diameter located near the midspan to ensure that the pressure distribution is symmetric on the two sides of the airfoil. To the purpose of this experiment, the model was equipped with trip strips to ensure a fixed transition location of the boundary layer. A zigzag tape was applied on both sides of the airfoil at $5 \%$ chord, see Figure 2 . The original trailing edge was modified by attaching trailing edge add-ons. For a baseline configuration, the extended pieces had a straight trailing edge. The different groups of students investigated different trailing edge geometry configurations; whose properties are listed in Table 1. The main extended panel at mid span was instrumented with several surface pressure microphones. 


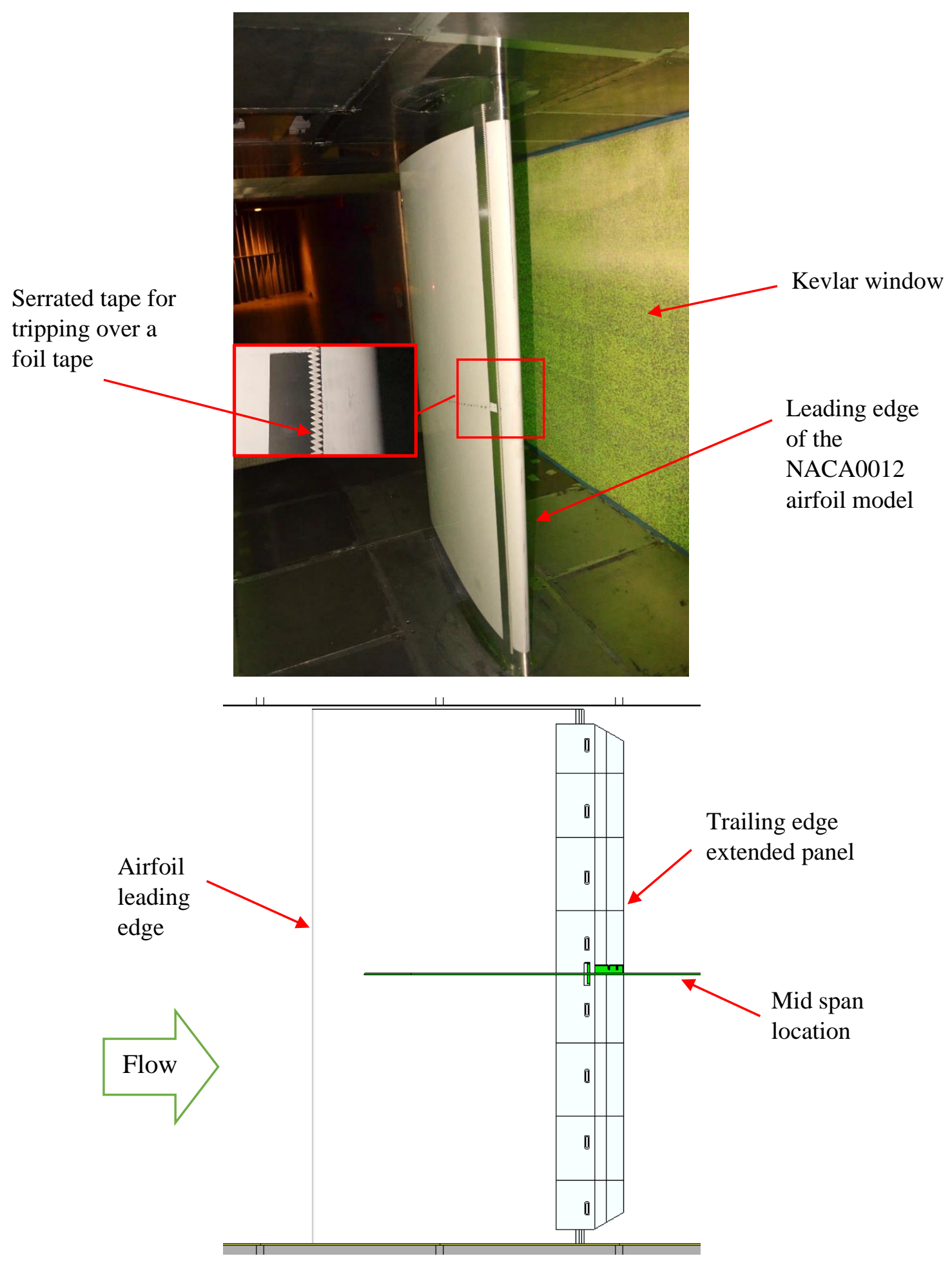

Figure 2: Top: A typical setup of the NACA0012 airfoil in the Stability Wind Tunnel as looking from upstream. Bottom: The schematic view of the NACA0012 airfoil with the straight edge add-on (baseline case). 


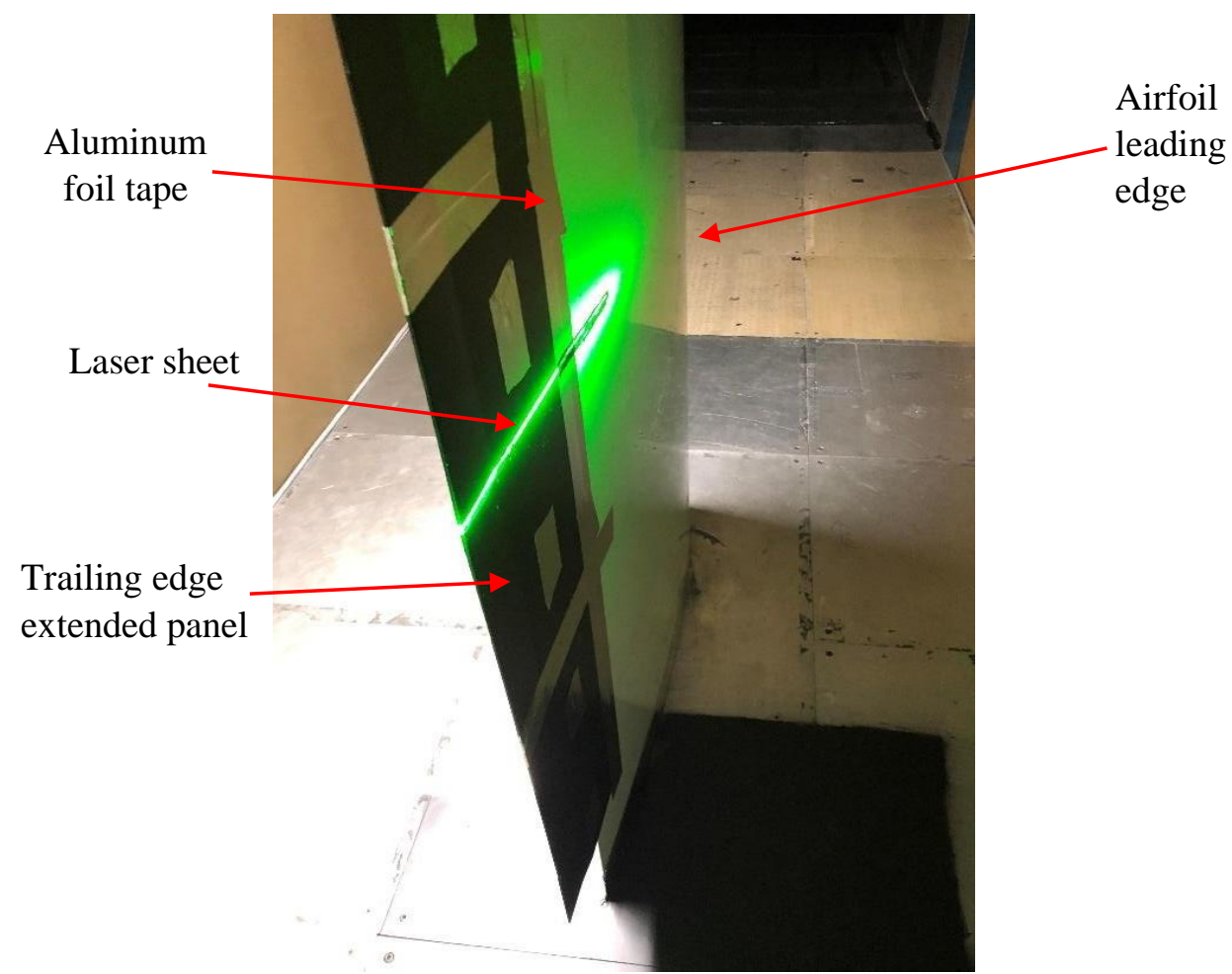

Figure 3: View of baseline extended panels as seen from downstream of the test section while the laser sheet is illuminated at the trailing edge.

\section{The trailing edge serrations}

Besides the baseline configuration, students investigated one of the 5 trailing edge serration cases listed in Table 1. All trailing edge add-ons were 3D printed, consisting of 7 spanwise panels, and were attached to the original trailing edge of the airfoil model. As seen in Figure 4 and Figure 5, the serrations consisted of 3 sawtooth and 2 slit configurations of varying dimensions. Two main parameters for the serration dimensions are the wavelength, $\lambda$, which is the distance between two serration teeth, and their tip-to-root height, $2 h$. For the slit configuration, another dimension, the slit width $(w)$ is introduced. The wavelength and slit width was kept constant while the tip-to-root height was varied to change the aspect ratio of the teeth, i.e. $2 h / \lambda$. List of dimensions for all 5 configurations can be found in Table 1. All configurations are equipped with surface microphones along the serrated edges as listed in the table. Sample diagram of the microphone locations (shown as brown dots in the figure) can be found in Figure 4 and Figure 5 . 


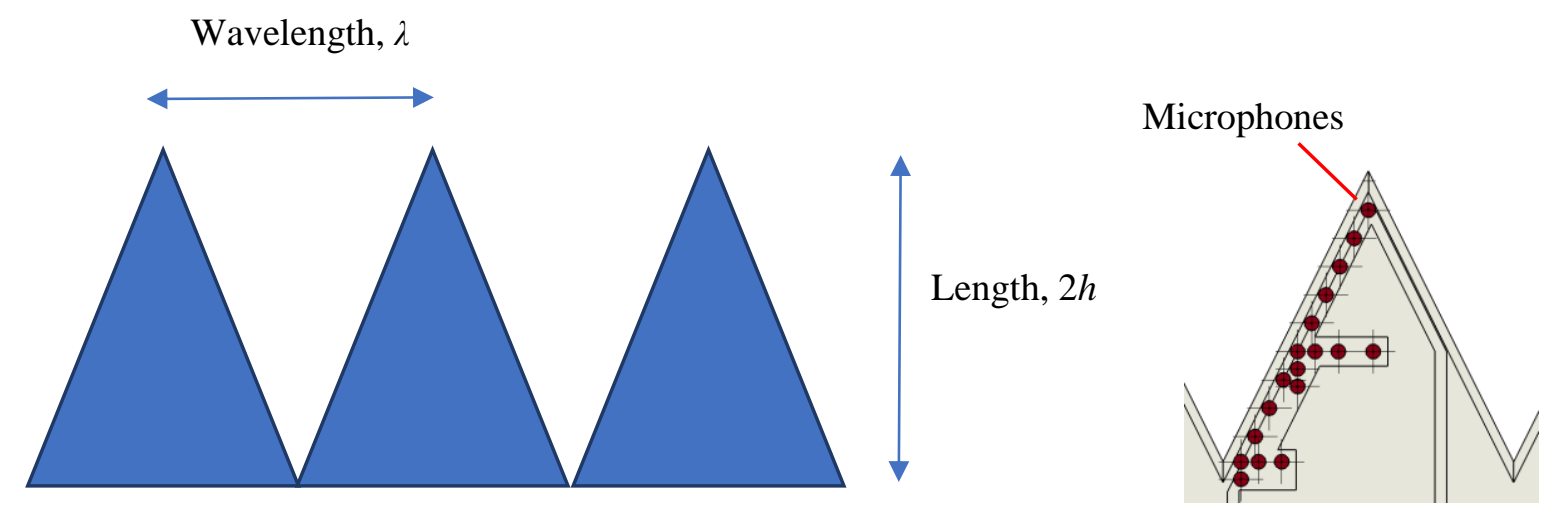

Figure 4: Geometry of the sawtooth serrations and diagram of surface microphone locations.
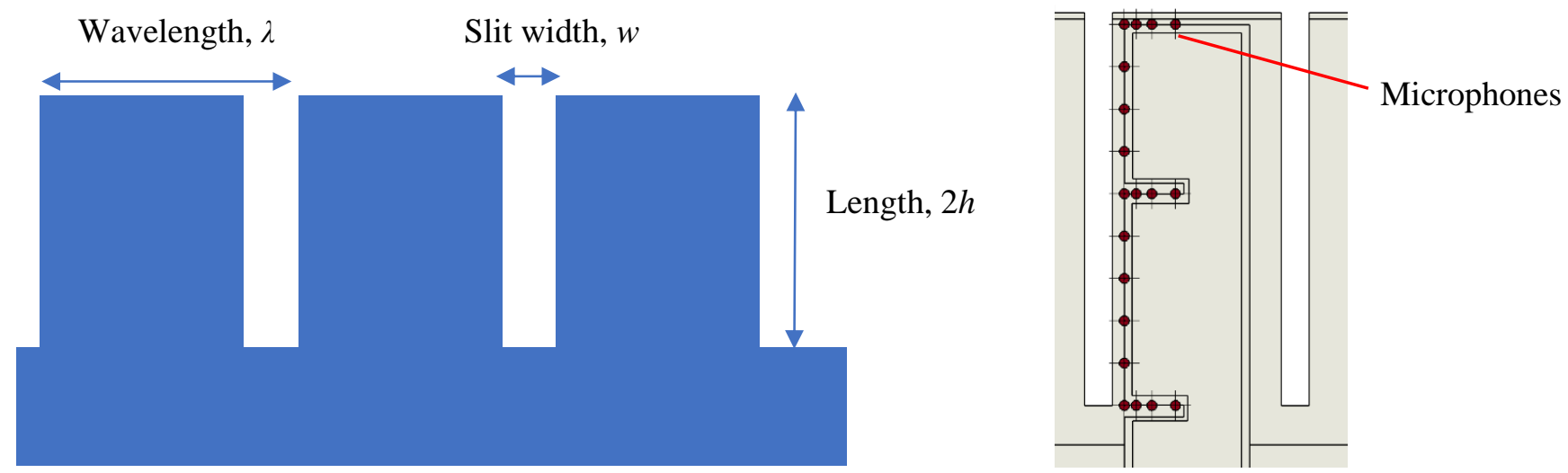

Figure 5: Geometry of the slit serrations and diagram of surface microphone locations.

Table 1: List of trailing edge configurations used in this experiment.

\begin{tabular}{|c|c|c|c|c|c|c|c|}
\hline $\begin{array}{c}\text { Serration } \\
\text { No. }\end{array}$ & $\begin{array}{c}\text { Serration } \\
\text { Type }\end{array}$ & $\begin{array}{c}\text { Wavelength } \\
(\lambda, \mathrm{cm})\end{array}$ & $\begin{array}{c}\text { Slit } \\
\text { width } \\
(w, \mathrm{~cm})\end{array}$ & $\begin{array}{c}\text { Tip-to- } \\
\text { root } \\
(2 h, \mathrm{~cm})\end{array}$ & $\begin{array}{c}\text { Aspect } \\
\text { Ratio } \\
(2 h / \lambda)\end{array}$ & $\begin{array}{c}\% \text { of } \\
\text { airfoil } \\
\text { chord }\end{array}$ & $\begin{array}{c}\text { Microphones } \\
\text { embedded } \\
(\text { edgewise/spanwise })\end{array}$ \\
\hline 1 & Sawtooth & 5 & - & 5 & 1 & 5 & $3 / 0$ \\
\hline 2 & Sawtooth & 5 & - & 10 & 2 & 11 & $5 / 3$ \\
\hline 3 & Sawtooth & 5 & - & 15 & 3 & 16.7 & $3 / 0$ \\
\hline 4 & Slit & 5 & 0.7143 & 5 & 1 & 5 & $3 / 0$ \\
\hline 5 & Slit & 5 & 0.7143 & 10 & 2 & 11 & $5 / 3$ \\
\hline 6 & $\begin{array}{c}\text { Baseline, } \\
\text { straight } \\
\text { edge }\end{array}$ & - & - & 10 & - & 11 & $5 / 3$ \\
\hline
\end{tabular}

\section{The particle image velocimetry system}

Besides the pressure fluctuation at the airfoil trailing edge and far field noise, a time-resolved PIV system was used to acquire the velocity field in the vicinity of the airfoil trailing edge. The PIV system in the Stability Wind Tunnel is a product of LaVision, which consists of 4 main components: (a) two high-speed Phantom v2512 cameras (but only one used in this setup), (b) a Photonics Industries DM150-532-DH type Nd:YAG dual head high-speed laser, 
(c) the programmable timing unit (PTU) and (d) the flow seeding unit. The PIV system offers the measurement of 2D and 3D velocity field within a $4.5 \mathrm{~mm}$ thick laser sheet at a maximum of $25.4 \mathrm{kFrames} / \mathrm{sec}$ sampling rate. The PTU is controlled through the LaVision Davis v10 software and is responsible for synchronizing the time instant of laser emission with the time instant of the camera acquiring the image of the illuminated particles.

As shown in Figure 3 and Figure 7, the PIV laser sheet was introduced inside the test section through a series of optics embedded within a vertical fairing of a NACA0018 shaped airfoil downstream of the airfoil trailing edge by approximately $0.7 \mathrm{~m}$ and $0.7 \mathrm{~m}$ off-center line. The camera was aligned with the surface of the serrations at a grazing angle, which helps to minimize the laser reflections off the surface. The field of view was approximately $12 \mathrm{~cm}$ by $7.5 \mathrm{~cm}$. The PIV system was used to obtain mean velocity data from the boundary layer, which was then provided to the students during the laboratory sessions in order to find boundary layer parameters, such as edge velocity and boundary layer thickness.

\section{Acoustic systems}

Two microphone systems were used in this study. The surface pressure fluctuations in the close vicinity of the trailing edge were measured using a series of miniature Knowles FG-23742-C05 type microphones embedded into the 3D printed trailing add-ons, see Figure 6. The microphone signals were sampled using Brüel and Kjaer (B\&K) LAN-XI data acquisition modules operated by a B\&K Pulse software. The gathered data was provided to the students during the laboratory sessions via a cloud service provider. Acoustic data was also gathered from the far field microphone array consisting of 251 microphones, see Figure 8. The purpose of this investigation was to obtain acoustic beamforming maps of the sound emitted by the airfoil to visualize the sound strength emitted by the airfoil trailing edge and the serration treatments. A signal of a single microphone from both the far field microphone array and the surface microphone array was provided to the students to Fourier transform the signal for further analysis.
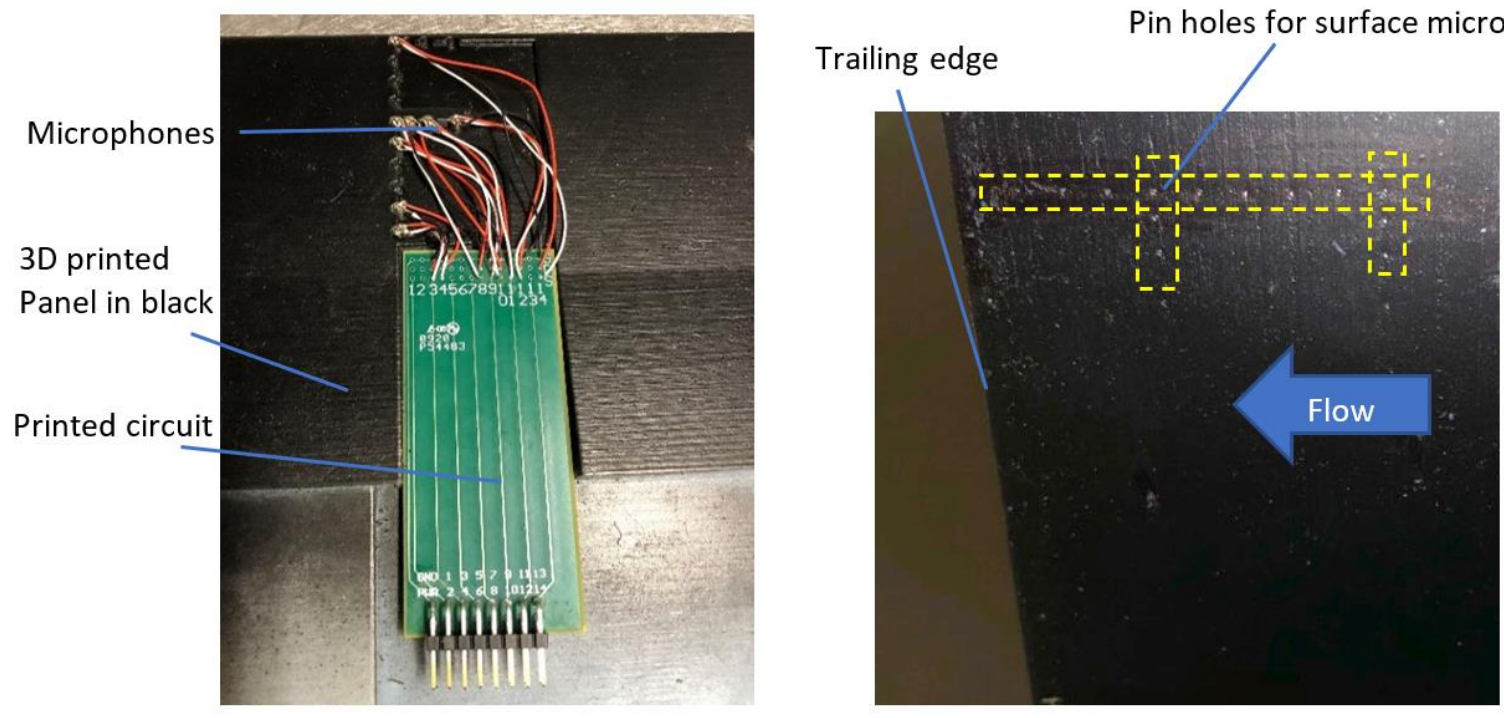

Figure 6: A picture of the surface pressure microphones embedded in the trailing edge addons (left) under a pinhole configuration (right). 


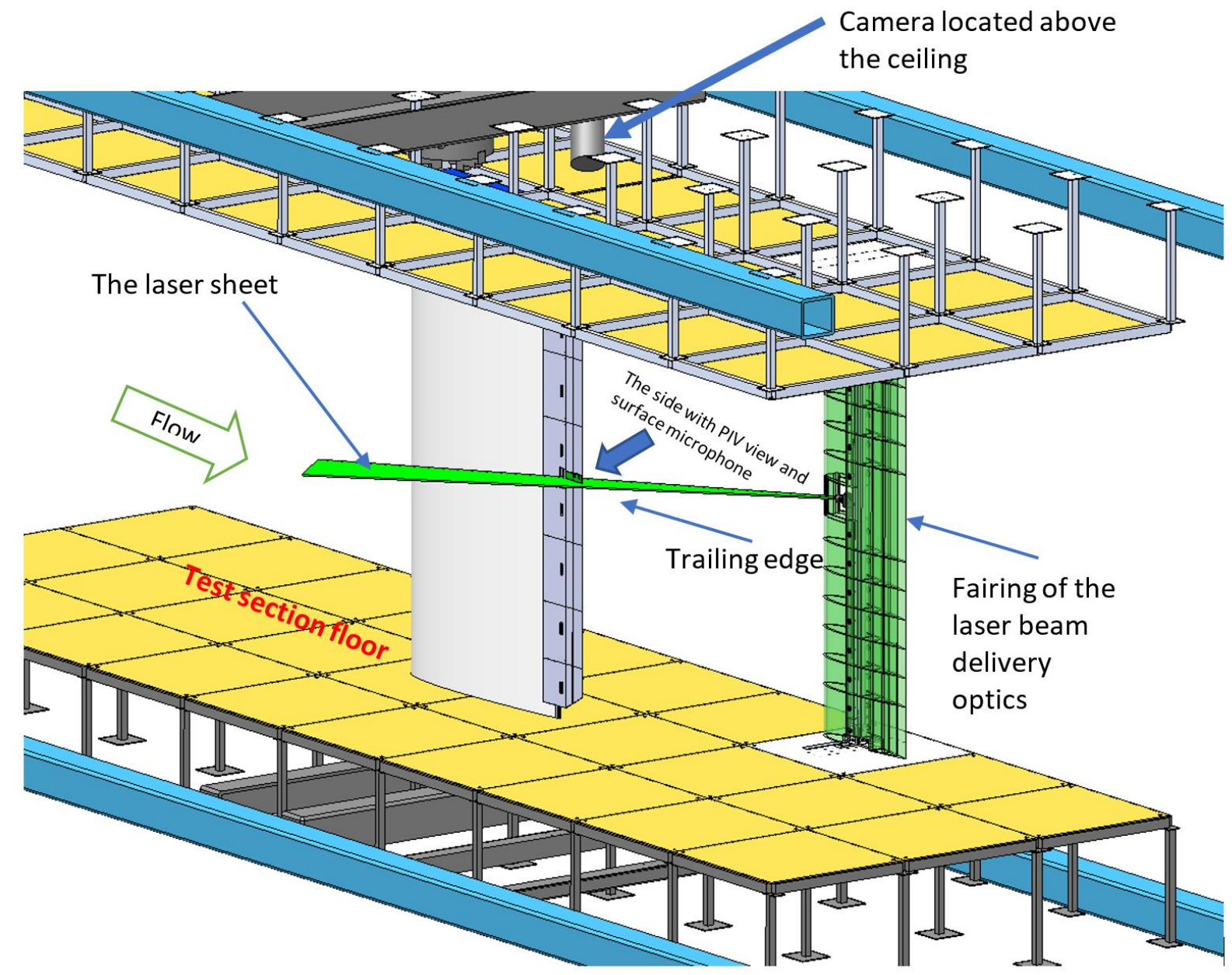

Figure 7: The schematic of the particle image velocimetry system.

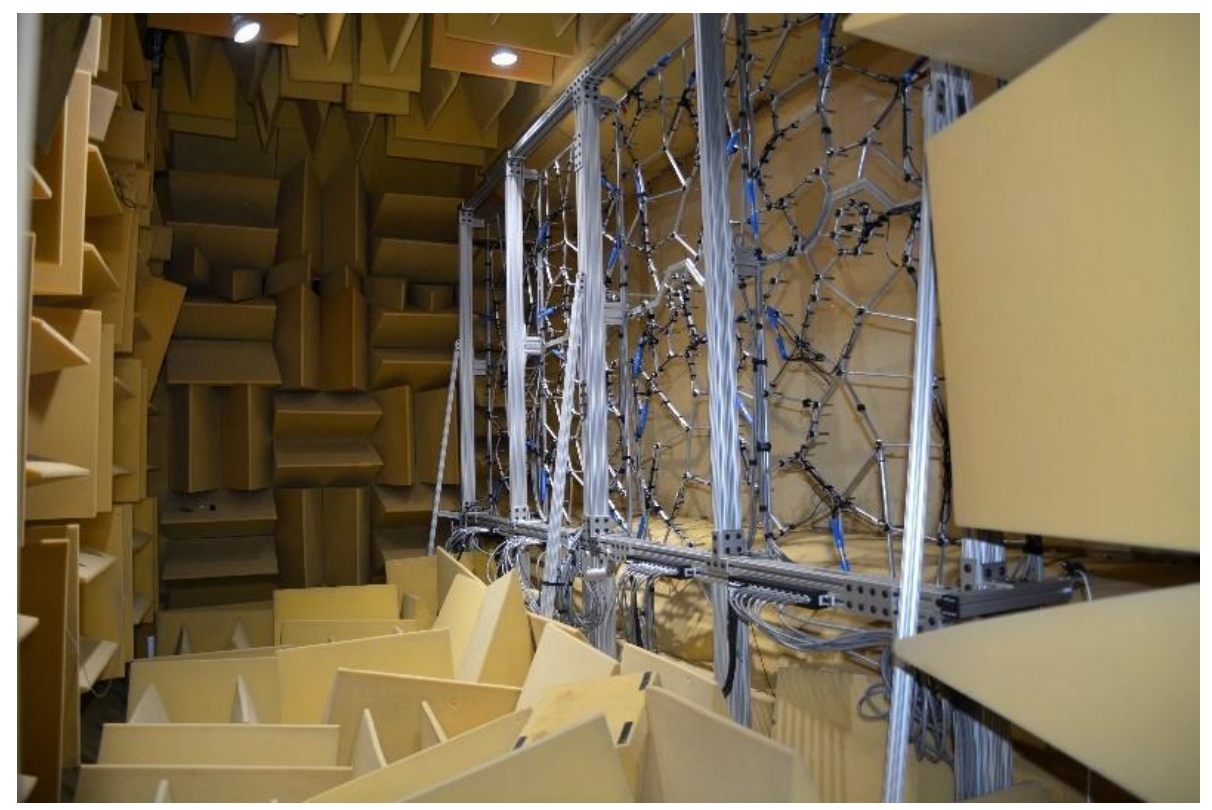

Figure 8: The Virginia Tech 251-channel microphone array located inside the starboard side anechoic chamber. 


\section{Components of the Remote Laboratory Sessions}

In the following, we review the components that were required to deliver the laboratory sessions to undergraduate students in a remote manner. To understand the requirements from each component, the structure of the labs alongside with the participants is introduced first. The laboratory sessions were split into 3 main parts, namely, each session began with (a) the tour of the facility followed by (b) data acquisition and (c) data processing and visualization. The laboratory sessions were conducted using the videoconferencing platform of Zoom to ensure social distancing. There was a maximum of six students (two groups of three) participating in every 2 hours long session alongside with two (on-site) tunnel personnel (tunnel operators) and an expert at a distance (faculty member or graduate student) who was also remotely present during the sessions. The schematic view of all participants is presented in Fig. 9. During the tour of the facility, the students were provided a walkthrough of the facility using a cell phone's camera handled by one of the tunnel operators. In this phase, the students were introduced to all equipment used in this study and they had the opportunity to interact with the tunnel personnel, while an expert at a distance kept the students engaged and evaluated their understanding of the experiments. The presence of the expert at a distance added key value to the laboratory sessions. During the tour, the students were responsible to document the experimental apparatus by capturing screenshots that served as input material for their laboratory reports. The students were also encouraged to record the video session on their own devices in support of their lab report writing. At the end of the tour, the expert at a distance left the video conference, and the tunnel operators began conducting the experiments. At the beginning of the data acquisition step, the screen sharing from all experimental apparatus, namely the wind tunnel camera system, the tunnel conditions, the surface microphone data acquisition software, the particle image velocimetry software, and the far field microphone array (beamforming array) software was turned on. These components are schematically shown in Figure 9.

During the data acquisition step, the students had the opportunity to visually engage with the experiments via the screen sharing function of the videoconference software. In each group, each student was assigned a different task: one person was responsible for logging the tunnel conditions (dynamic pressure, temperature, static pressure, flow speed, etc.) at all measurement points; one person was responsible for obtaining screenshots from the live videoconference session in support of their laboratory report; and the third student in each group was responsible for pre-processing the data. During the data acquisition step, a ramp-shaped surface add-on was installed on the surface of the airfoil by one of the tunnel operators. The students, working as a team, were responsible to identify a location of the surface add-on from a set of options (streamwise location and orientation) that will significantly enhance the generated trailing edge noise. This step challenged their understanding of the underlying physics while it also provided them a possibility to interact with the wind tunnel experiment. After the installation of the surface add-on, acoustic data was gathered with the presence of the surface add-on. This measurement concluded the data acquisition step. The gathered data was then exported from the acquisition software and it was made available to the students via a cloud service. The students received a URL link to the cloud from where they could immediately download the data for further processing.

At the last phase, the data processing and visualization phase, the students were provided results processed by each individual data acquisition system of the various experimental apparatus, such as the far field, surface pressure microphone array, and PIV. In this step, the tunnel operators interpreted the results to the students while they had the opportunity to engage in the discussion with the tunnel personnel. 


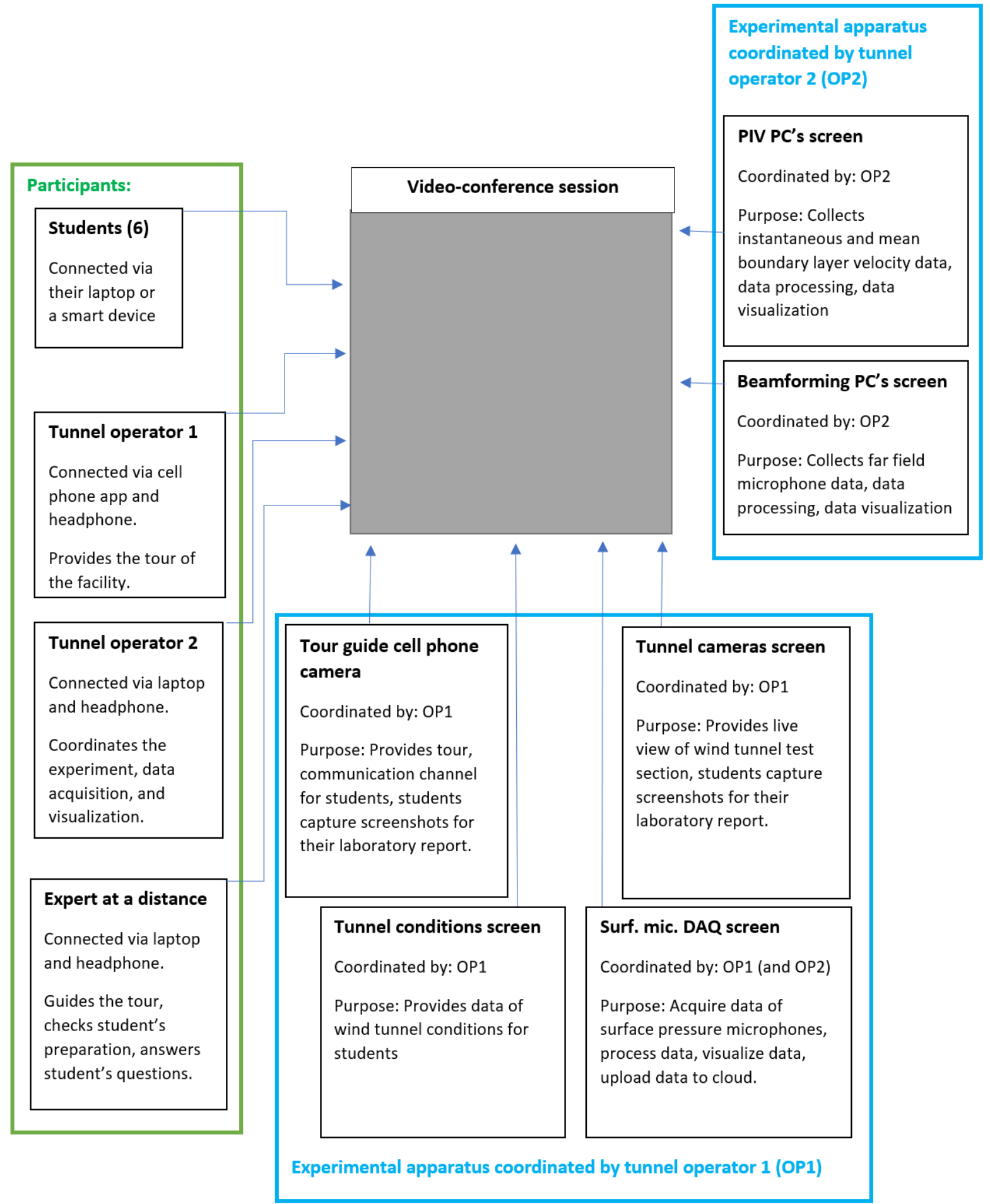

Figure 9: Components of the laboratory sessions. 


\section{Laboratory evaluation}

\section{A. Evaluation metrics}

At the end of the two-week long experimental campaign, the students were asked to answer a questionnaire tailored to measure their experience during the remote laboratory sessions. The purpose of the questionnaire was to evaluate the efficiency of the remote laboratories. A total number of 175 students were enrolled in the class, and 170 responses were collected using the questionnaire. While filling the questionnaire was voluntary and optional, it was offered as an extra assignment for credit within the course. The questionnaire consisted of two parts. In the first part, students were provided statements (closed-ended questionnaire) to which they were asked to respond by choosing from the options of (5) Strongly Agree, (4) Agree, (3) Neutral, (2) Disagree, (1) Strongly Disagree. These closed-ended questions were then followed by open-ended questions in the second part of the questionnaire. The closed-ended questions targeted three main categories, namely, the (a) students' experience during the labs, (b) the students' interaction experience with the various components (see Fig. 9.) of the lab sessions and (c) the students' overall impressions. In the following, we present the statements within each category.

The following statements were used to evaluate the students' experience during the laboratory sessions:

- I felt the wind tunnel staff made me feel involved with the tour of the facility.

- The wind tunnel tour gave me a good sense of the scale of the facility used in this lab.

- I am satisfied that I saw all the features and aspects of the facility that I was interested in.

- The expert at a distance kept me engaged throughout the wind tunnel tour.

- The expert at a distance answered all my questions.

- At the end of the tour, I had a clear picture of the various equipment that were to be used during the laboratory session.

- The data visualization and its interpretation were clear and easy to follow.

The efficiency of student-instructor and student-teleconferencing session interactions were evaluated using the following statements:

- The Zoom navigation video provided before the lab adequately prepared me for using the various functionalities during the lab.

- The Zoom session was easy to navigate and it was clear what computer screens I had available for use.

- I felt the research staff made me feel involved in the measurements.

- I felt like I had some level of control of the experiments.

- Generally, it was clear and easy to understand what is happening in this experiment.

- I felt the research staff made me feel involved with the data processing.

- $\quad$ Upon completion of the experiment, my data sets were made available to me in a timely manner.

Finally, the questionnaire asked the students to provide their overall impressions by responding to the following statements:

- At the end of the experiment, it was clear to me what measurement apparatus had been used and what type of data each collects.

- Generally, I felt the wind tunnel staff made me feel involved with all aspects of the experiment.

- Thanks to this experiment, I have a clear sense of what it takes to run a wind tunnel test in a large-scale facility.

- $\quad$ Overall, I enjoyed this lab.

- I would be glad to perform more experiments using such remote setup. 


\section{B. Results}

Figure 10 presents students' experiences during the laboratory sessions. Results are shown as the percentage of student feedback per statement. Overall, the results reveal that the student's experience during the labs was strongly positive as in the majority of the responses (70-90\%) students either agreed or strongly agreed with the presented positive statements. The lack of students' physical presence can significantly compromise their laboratory experience. This drawback of the remote labs was successfully addressed with the tour of the facility while the interactions between the students and instructors (expert at a distance and wind tunnel staff) also added key value to the sessions. The SWT is a rather large facility on the scale of university-based wind tunnel facilities. Based on students' feedback, the tour of the facility provided a good estimate of the scales of the facility to the students.

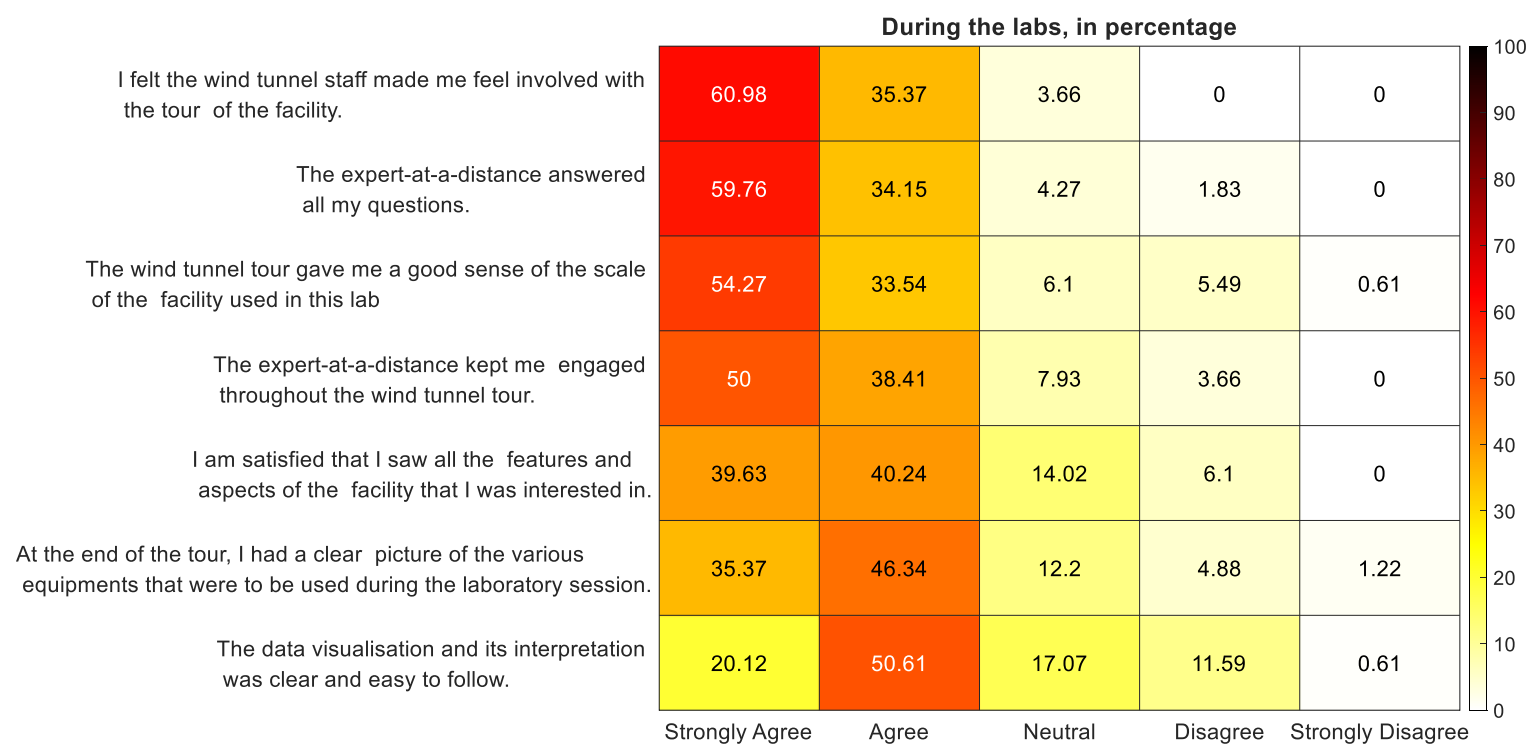

Figure 10: Results of the closed-ended questionnaire: Students experience during the labs.

While the tour of the facility was proven to be a successful way to overcome students' lack of physical presence in the facility, the interaction between the students and instrumentation was a more challenging task to resolve because the direct control of the instrumentation could not be made available to the students due to the short period of time available for laboratory preparation. Figure 11 presents students' feedback on the interaction-specific statements of the closed-ended questionnaire. Overall, the responses were still positive on average, with most of the responses still observed under the agree or strongly agree categories. Based on the responses, uploading files to the cloud was an efficient way to transfer the acquired data to the students. Students were provided a video tutorial prior to the labs focusing on how to navigate and operate within the Zoom teleconferencing software. Their feedback suggests that they seemed to observe some difficulties using the software. A possible explanation for this is that when the laboratory sessions were conducted (early April 2020), the Zoom teleconferencing software was relatively new to the entire academic community and students were still in the adopting phase. Similarly, the evaluation of the data acquisition process and data visualization process was positive and most of the responses were observed in the agree category. The possible reasons for the drop in the percentage of students strongly agreeing can be explained with the difficulty of navigating within the Zoom sessions and the lack of direct student control over the instrumentation. The latter is clearly indicated by the responses observed to the last statement of Fig. 11, where the level of students' control over the experiments was targeted. While some control could be given to the students, for example during the installation of the surface add-on, they still seem to prefer an entirely remotely controlled experiment. Due to the lack of time and the large-scale nature of the facility used here the complete remote control of the instrumentation could not be made available to the students for this laboratory. 


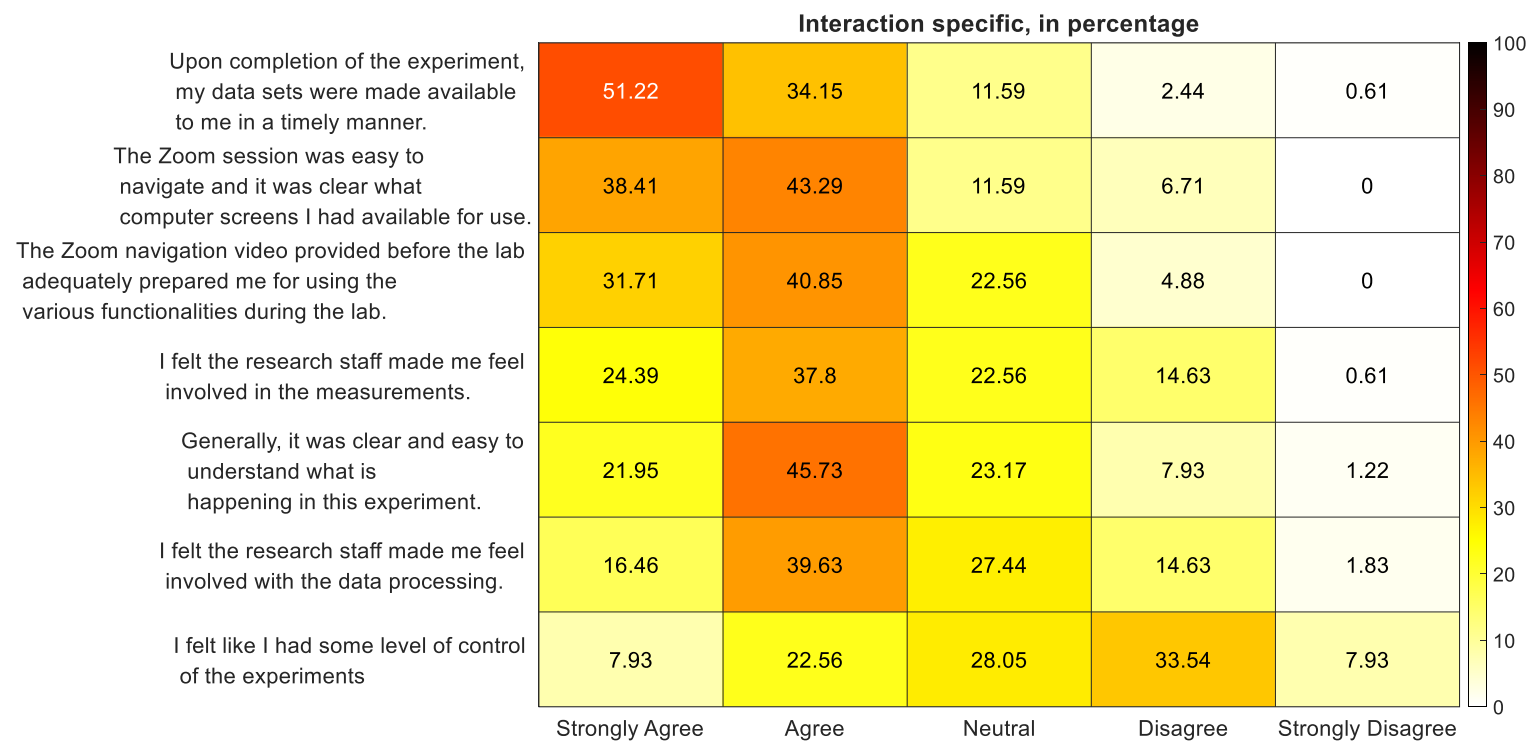

Figure 11: Results of the closed-ended questionnaire: Interaction specific metrics.

Finally, Fig. 12 presents the student's overall impressions. While the majority of the feedback is still rather positive, some shift to the disagree and strongly disagree responses can be observed. Based on the results of Fig. 12, students reported to have a general understanding of what experimental apparatus was used, they felt involved in the experiments and they also reported to gain a sense of what it takes to perform wind tunnel experiments at a large scale facility. In contrast, the last statement presented in Fig. 12 received more wide-spread responses. These feedbacks were most likely based on the interpretation of the statement. Considering the pandemic situation, remote experiments are clearly a far superior alternative to canceling the laboratory sessions. In this interpretation, the students were presumably more likely to favor a remote laboratory session. On the other hand, at the time of the questionnaire (April 2020), many assumed that the fall semester of 2020 would be conducted in a regular, in person delivery fashion. Under regular circumstances, students most likely would prefer the in-person laboratory sessions presented here. Overall, the remote labs discussed in this paper can be considered a successful and efficient way to ensure the laboratory delivery of wind tunnel experiments to the students.

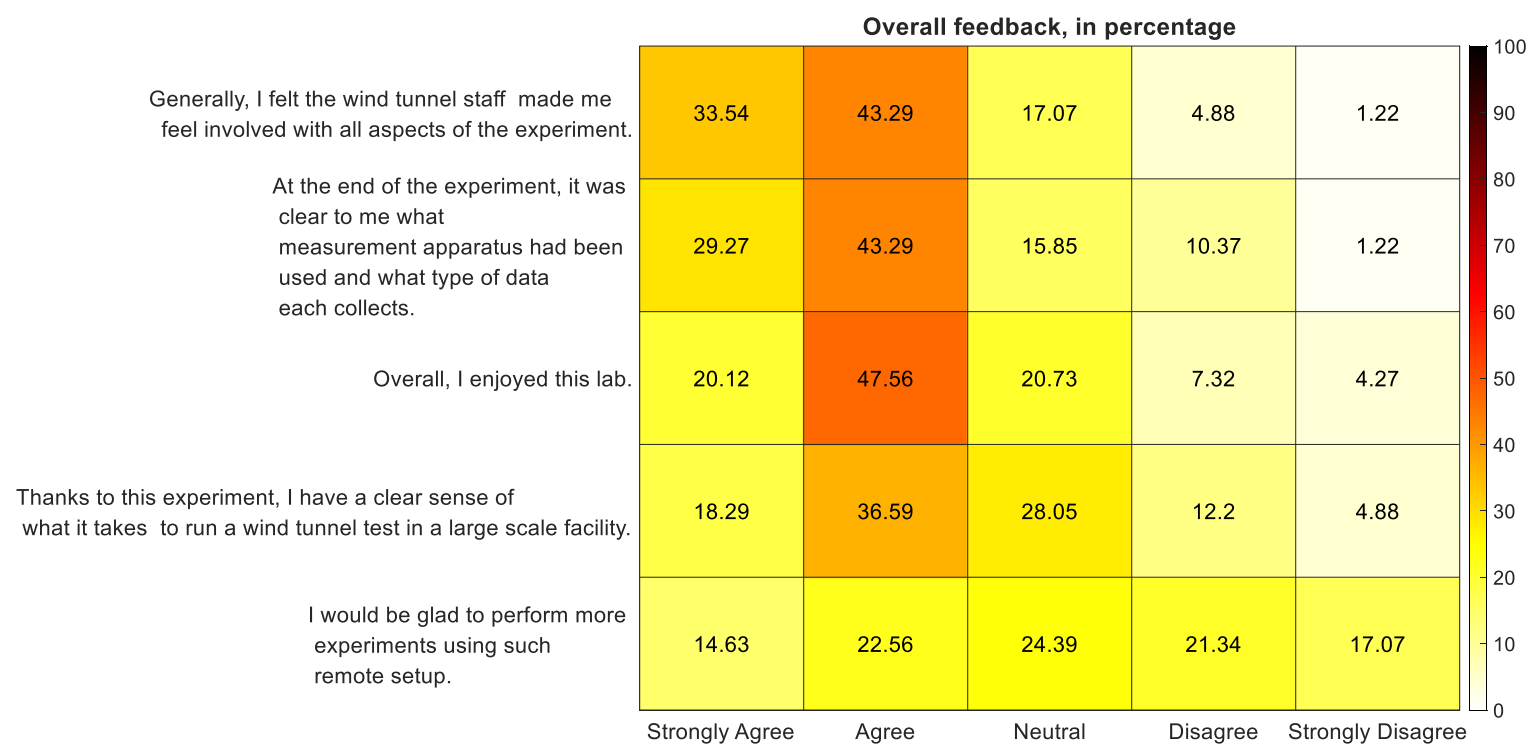

Figure 12: Results of the closed-ended questionnaire: Students overall impressions. 
In addition to the closed-ended questions, a comparison is made between the past five-year average (2014-2019) of the student lab report scores and the student lab report scores of 2020. While previous labs were in-person, they also comprised of a different experiment each year. While this is not a rigorous comparison of in-person vs. remote labs, comparing the remote labs of 2020 to a wide range of labs covered by the historical data provides a general evaluation of student performance under various conditions.

Student lab report scores of the past five years (2014-2020) and the lab report scores of the remote labs discussed in this paper are presented in Fig. 13. The lab report scores are presented as a histogram in 10\%-wide bands. The results reveal that the remote labs show a similar score distribution to the average of the previous five-year lab scores. The student lab report scores of the remote labs are also very close to the five-year average in all bands and only marginal differences can be observed.

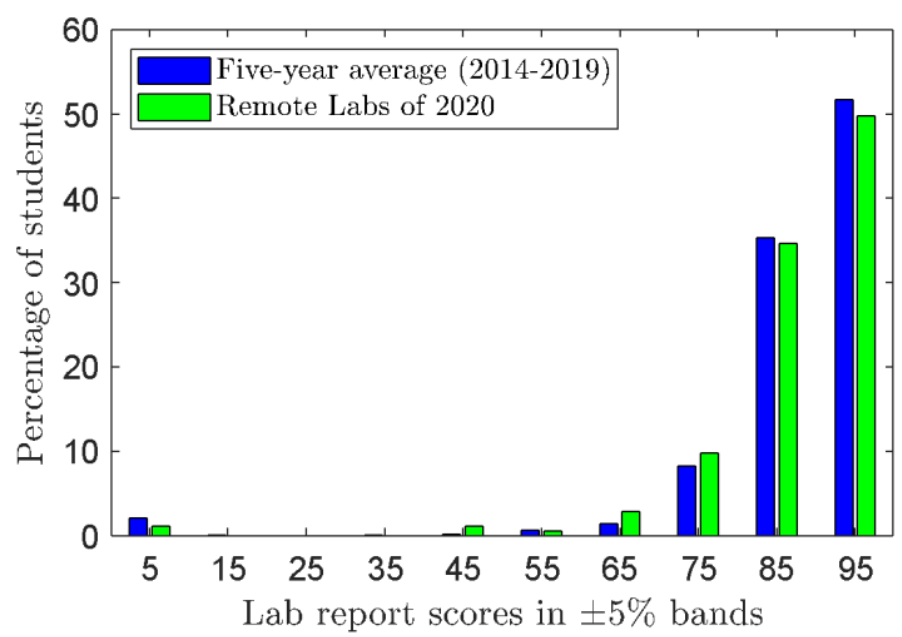

Figure 13: Students laboratory report scores compared to five-year average (2014-2015). 


\section{V.Summary}

This paper presents the remote delivery of laboratory experiments performed during the early stages of the coronavirus pandemic. The labs presented here were conducted during April 2020 at the Stability Wind Tunnel of Virginia Tech and they were originally scheduled as in-person laboratory sessions. The laboratory sessions assessed the aeroacoustic noise reduction efficiency of various trailing edge serrations on the generated trailing edge noise of a NACA0012 airfoil. In their laboratory reports, students were responsible to perform a Fourier analysis of the gathered acoustic data. After the laboratory sessions, some 170 students provided feedback on the efficacy of the laboratory sessions via a questionnaire comprising open-ended and closed-ended questions.

All necessary components that ensured the successful remote delivery of the laboratory sessions are presented and discussed in detail. A widely available and commonly used teleconferencing software was used to stream the screens of all instrumentation to the students, while a set of cameras provided a visual immersion of the students to the facility. Each laboratory session consisted of three main parts, namely, tour of the facility, data acquisition and data visualization. During the tour of the facility, an expert at a distance (faculty member or graduate student) was also present in the teleconferencing session and interacted actively with the students to ensure they have a good understanding of the underlying physics and they gain a clear view of the experiment. During the data acquisition and data visualization process, the students closely followed the displays of all instruments used during the experiments. Finally, the acquired data was made available to the students via a cloud service. The gathered data was also visualized and discussed at the end of each laboratory session.

Students' feedback via the open-ended questionnaire revealed that the remote delivery method of the laboratory sessions presented here is an efficient and successful way to perform undergraduate laboratory experiments during the coronavirus pandemic. The results from the open-ended questionnaire confirmed that the tour of the facility provided a clear sense of the scale of the facility, and the instrumentation used in the experiment. The teleconferencing session was also proven to be a sufficient method to deliver the screens of various instruments to the students. Overall, students reported that they were satisfied with the laboratory sessions, but the responses also revealed that their preference is still biased toward in-person laboratory sessions.

The present study demonstrates that standard video conferencing tools are already sufficient for effective delivery of remote experiments in an educational setting. It also demonstrates that such experiments are perfectly practical in a large scale facility and, indeed, in some ways improve upon the experience that can be gained in an in-person setting. There is great scope for expansion here. Properly designed remote laboratory experiences have the potential to place unique and internationally significant facilities in the hands of students and professionals wherever they are located. This can be performed at comparatively low cost and in a setting that preserves the security and integrity of the facility while eliminating the geographic and economic barriers that ordinarily prevent such sharing.

\section{Acknowledgements}

The work presented in this paper could not be possible without the support of a large set of people. The authors would like to thank the support of the laboratory delivery during the unprecedented times of the COVID-19 pandemic to the following personnel. We would like to thank Bill Oetjens for supporting the preparation and the delivery of the remote laboratories. We would like to thank Vidya Vishwanathan, Aldo Gargiulo, Julie Duetsch-Patel, Danny Fritsch, Christopher Hickling and Agastya Balantrapu for serving as experts at a distance during the laboratory tours. We also want to thank Dr. Lorna Ayton and Dr. Chaitanya Paruchuri for providing pre-recorded lectures on frequency domain analysis and trailing edge noise generation. We greatly appreciate the support from Virginia Tech College of Engineering and Virginia Tech Department of Aerospace and Ocean Engineering. 


\section{References}

[1] M. Ogot, G. Elliott, and N. Glumac, "Hands-on laboratory experience via remote control: Jet thrust laboratory," ASEE Annu. Conf. Proc., pp. 10357-10374, 2002.

[2] G. C. Goodwin, A. M. Medioli, W. Sher, L. B. Vlacic, and J. S. Welsh, "Emulation-based virtual laboratories: A low-cost alternative to physical experiments in control engineering education," IEEE Trans. Educ., vol. 54, no. 1, pp. 48-55, 2011, doi: 10.1109/TE.2010.2043434.

[3] S. Lal et al., "Student perceptions of instruction sheets in face-to-face and remotely-operated engineering laboratory learning," Eur. J. Eng. Educ., vol. 45, no. 4, pp. 491-515, 2020, doi: 10.1080/03043797.2019.1654433.

[4] J. Ma and J. V. Nickerson, "Hands-on, simulated, and remote laboratories: A comparative literature review," ACM Comput. Surv., vol. 38, no. 3, p. 1, 2006, doi: 10.1145/1132960.1132961.

[5] J. V. Nickerson, J. E. Corter, S. K. Esche, and C. Chassapis, "A model for evaluating the effectiveness of remote engineering laboratories and simulations in education," Comput. Educ., vol. 49, no. 3, pp. 708-725, 2007, doi: 10.1016/j.compedu.2005.11.019.

[6] I. A. Clark et al., "Bioinspired trailing-edge noise control," AIAA J., vol. 55, no. 3, pp. 740-754, 2017, doi: 10.2514/1.J055243.

[7] H. H. Murray, W. J. Devenport, W. N. Alexander, S. A. L. Glegg, and D. Wisda, "Aeroacoustics of a rotor ingesting a planar boundary layer at high thrust," J. Fluid Mech., vol. 850, pp. 212-215, 2018, doi: $10.1017 / \mathrm{jfm} .2018 .438$.

[8] W. N. Alexander, W. J. Devenport, and S. A. L. Glegg, "Noise from a rotor ingesting a thick boundary layer and relation to measurements of ingested turbulence," J. Sound Vib., vol. 409, pp. 227-240, 2017, doi: 10.1016/j.jsv.2017.07.056.

[9] K. Brown, J. Brown, M. Patil, and W. Devenport, "Inverse measurement of wall pressure field in flexiblewall wind tunnels using global wall deformation data," Exp. Fluids, vol. 59, no. 2, p. 0, 2018, doi: 10.1007/s00348-017-2477-9.

[10] L. A. Joseph, N. J. Molinaro, W. J. Devenport, and T. W. Meyers, "Characteristics of the pressure fluctuations generated in turbulent boundary layers over rough surfaces," J. Fluid Mech., vol. 883, 2020, doi: $10.1017 / \mathrm{jfm} .2019 .813$.

[11] T. F. Brooks, D. S. Pope, and M. A. Marcolini, "Airfoil self-noise and prediction," NASA Ref. Publ., no. $1218,1989$.

[12] R. K. Amiet, "Noise due to turbulent flow past a trailing edge," J. Sound Vib., vol. 47, no. 3, pp. 387-393, 1976, doi: 10.1016/0022-460X(76)90948-2.

[13] VT News, “Aerospace engineering labs continue on through faculty's creative approach,” 2020. https://vtnews.vt.edu/articles/2020/05/aoe-remote-experimentation.html Accessed on 11/24/2020 